\title{
CONSTRUCTION OF AN E-CALLISTO STATION IN KOREA
}

\author{
Su-Chan Bong ${ }^{1}$, Yeon-Han Kim ${ }^{1}$, HeeSeon Roh ${ }^{2}$, Kyung-Suk Cho ${ }^{1}$, Young-Deuk Park ${ }^{1}$, Seonghwan Choi ${ }^{1}$, \\ Ji-Hye Baek ${ }^{1}$, Christian Monstein ${ }^{3}$, Arnold O. Benz ${ }^{3}$, Yong-Jae Moon ${ }^{2}$, and Sungsoo S. Kim ${ }^{2}$ \\ ${ }^{1}$ Korea Astronomy and Space Science Institute, 61-1 Hwaam-dong, Yuseong-gu, Daejeon, 305-348, Korea \\ E-mail: scbong@kasi.re.kr \\ ${ }^{2}$ Department of Astronomy and Space Science, Kyung Hee University, Yongin-si, Gyeonggi-do, 446-701, Korea \\ ${ }^{3}$ Institute of Astronomy, ETH Zurich, CH-8093 Zurich, Switzerland \\ (Received December 4, 2008; Accepted January 20, 2009)
}

\begin{abstract}
The e-CALLISTO is a global network of frequency-agile solar radio spectrometers that was constructed in a collaboration between Swiss Federal Institute of Technology Zurich (ETH Zurich) and local host institutes. It is intended to monitor solar radio bursts 24 hours a day in frequency range between $45 \mathrm{MHz}$ and $870 \mathrm{MHz}$. One of e-CALLISTO spectrometer was installed at Korea Astronomy and Space Science Institute (KASI) in 2007 October. The spectrometer gets signals from a horizontally polarized log-periodic antenna mounted on an automatic Sun-tracking system. Tracking status and data are monitored in Space Weather Monitoring Laboratory (SWML) of KASI in real time, and flare time data are transferred to ETH Zurich data archive daily. Using this spectrometer we obtained a couple of type II solar radio bursts on 2007 December 31, and found that these bursts are associated with a CME which occurred on the east limb.
\end{abstract}

Key words : instrumentation: spectrographs — Sun: coronal mass ejections — Sun: radio radiation

\section{INTRODUCTION}

Solar radio bursts, which often accompany flares and coronal mass ejections (CMEs), occur in many varieties over wide frequency range from $\mathrm{MHz}$ to tens of $\mathrm{GHz}$ with large variability of amplitudes. In the metric wavelength range around $100 \mathrm{MHz}$, they are classified into five different types based on spectrum and temporal behavior (Wild 1985). In the decimetric wavelength range around $1 \mathrm{GHz}$, they are also classified into another five different types (Isliker \& Benz 1994).

Since their discovery in 1942 through the interference with radar systems (Hey 1946), the understanding and prediction of solar radio bursts have been of considerable interest. In view of space weather implication, excess radio noise produced by strong solar radio bursts has impacts on geosynchronous satellite transmissions, wireless cell sites, Global Positioning Satellite (GPS) signals, and radars (Lanzerotti 2004; Cerruti et al. 2008). Also in view of scientific diagnosis of the events, radio waves at meter and decimeter waves can provide information on shocks, energetic electrons, and their acceleration (Gary \& Hurford 2004). Especially, type II radio bursts, which are regarded to be the signatures of the shock-associated plasma oscillations, have been intensively studied to understand their sources and in connection with CMEs and solar energetic particle (SEP) events (Gopalswamy et al. 2005; Cho et al. 2005, 2007, 2008; Shen et al. 2007; Evans et al. 2008).
However, many aspects of various radio emissions are still largely unknown after more than 60 years of study.

The radio spectrometer, CALLISTO (Compact Astronomical Low-cost Low-frequency Instrument for Spectroscopy and Transportable Observatory; Benz et al. 2005,2009 ), is a frequency agile receiver developed by Swiss Federal Institute of Technology Zurich (ETH Zurich), based on commercially available consumer electronics. It features the low price $(<200$ USD), small size $(110 \mathrm{~mm} \times 80 \mathrm{~mm} \times 205 \mathrm{~mm})$, and light weight $(\sim 800 \mathrm{~g})$. The allowed measuring frequency ranges from $45 \mathrm{MHz}$ to $870 \mathrm{MHz}$, covering meter and low decimeter band, in steps of $62.5 \mathrm{kHz}$. The CALLISTO spectrometers distributed around the globe form a network called e-CALLISTO. The e-CALLISTO network was initialized in the frame of the International Heliospheric Year (IHY; Davila et al. 2004) announced by the United Nation Organization (UNO) for 2007. The spectrometer unit is provided by ETH Zurich, and the others including antenna and PC interface to the internet are provided by the local host institutes. It aims at 24 hour coverage of the radio emission of the Sun. Currently 11 stations are operational worldwide and the number is still growing as redundancy is desirable.

Here we report on the construction of Korean eCALLISTO station at Korea Astronomy and Space Science Institute (KASI). System configuration is described in $\S 2$, test in $\S 3$, and first results in $\S 4$. We summarize and discuss the result in $\S 5$.

Corresponding Author: S.-C. Bong 


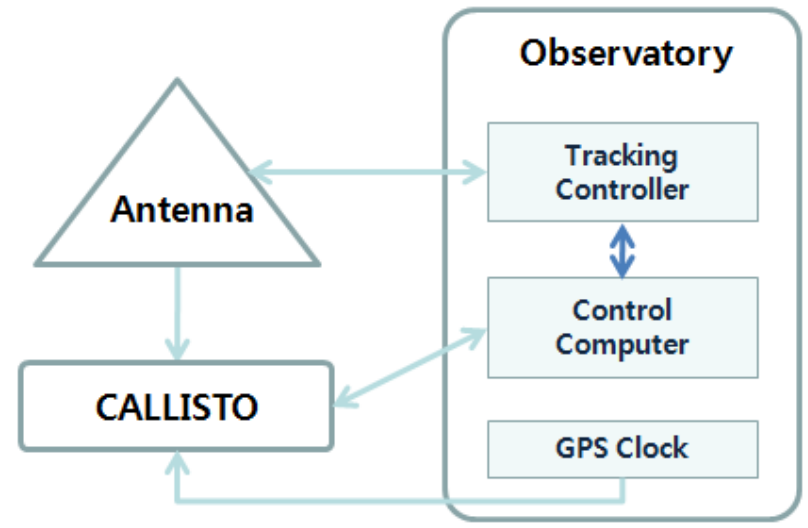

Fig. 1.- Schematic diagram of the observation system. The main observation system consists of an antenna, a CALLISTO spectrometer, and a Windows computer connected to the internet. In addition, a GPS clock controls the sampling time of the spectrometer, and a tracking controller controls the antenna direction.

TABLE 1.

Geographical Coordinates of the Site

\begin{tabular}{ll}
\hline Coordinate & Value \\
\hline Latitude & $36^{\circ} 23^{\prime} 50^{\prime \prime}$ North \\
Longitude & $127^{\circ} 22^{\prime} 31^{\prime \prime}$ East \\
Height & $120 \mathrm{~m}$ \\
Local Time & UTC $+09 \mathrm{~h}$ \\
\hline
\end{tabular}

\section{SYSTEM CONFIGURATION}

The main observation system consists of an antenna, a CALLISTO spectrometer, and a Windows computer connected to the internet. In addition, a GPS clock controls the sampling time of the spectrometer, and a tracking controller controls the antenna direction. Fig. 1 shows the schematic diagram of the observation system.

The site is located in the KASI headquarters which is in the suburb of Daejeon, Korea. The antenna was constructed between the $14 \mathrm{~m}$ radio telescope of Taeduk Radio Astronomy Observatory (TRAO; Roh \& Jung 1999) and KASI Solar Imaging Spectrograph (KSIS; Kim et al. 2006). Table 1 summarizes the geographical coordinates of installation site.

We use a log periodic dipole antenna directly connected via a low loss coaxial cable to the CALLISTO spectrometer (Fig. 2). The antenna LP-1018 has a boom length of $5 \mathrm{~m}$ and covers the range from $30 \mathrm{MHz}$ up to $1100 \mathrm{MHz}$ with gain $8-9 \mathrm{dBi}$. The average full width at half maximum (FWHM) is $65^{\circ}$ in the electric field (E) plane and $110^{\circ}$ in the magnetizing field $(\mathrm{H})$ plane.

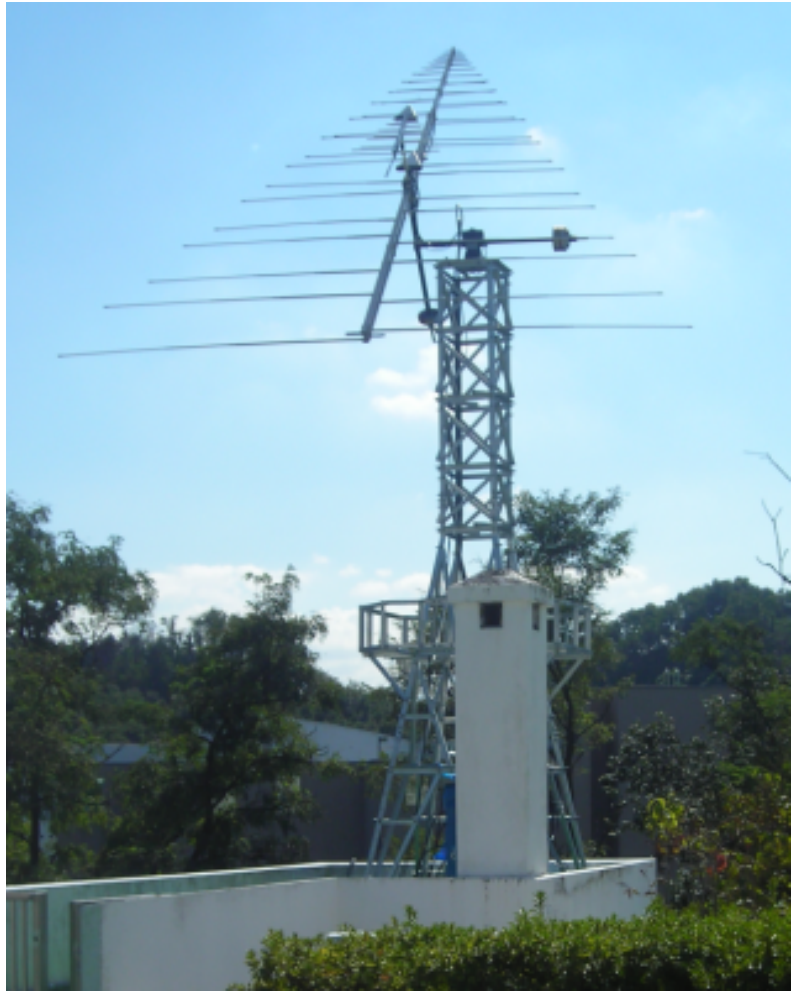

Fig. 2.- The log periodic dipole antenna of the station. It is mounted horizontally on an azimuth/elevation mount on top of a $6 \mathrm{~m}$ high tower. CALLISTO receiver is located in a steel case underneath the tower and remotely controlled from the nearby observatory using RS-422 link.

The antenna is mounted horizontally on an azimuth/elevation mount on top of a $6 \mathrm{~m}$ high tower. The antenna mount and tracking control system is designed to automatically direct the antenna to the Sun with $\sim 1^{\circ}$ precision during day time. The control software calculates the current Sun position based on the current computer time and rotates the antenna to the Sun if the elevation of the Sun is greater than or equal to $10^{\circ}$. Current positions of the Sun and the antenna are recorded every minute to a daily log file for monitoring. The computer time is automatically synchronized to a public Network Time Protocol (NTP) time server every hour.

The signal from the antenna is directed to the CALLISTO spectrometer, which is housed in a steel case underneath the tower, via a low loss coaxial cable, LMR240. It is $12 \mathrm{~m}$ long so that the cable attenuation at 50 , 450 , and $900 \mathrm{MHz}$ is $0.7,2.1$, and $3.0 \mathrm{~dB}$, respectively. The CALLISTO spectrometer having a detector sensitivity of $25 \mathrm{mV} / \mathrm{dB}$ was supplied by ETH Zurich. The frequency range of CALLISTO ranges from $45 \mathrm{MHz}$ to $870 \mathrm{MHz}$ in three sub bands. The channel resolution is $62.5 \mathrm{kHz}$, while the radiometric bandwidth is about 


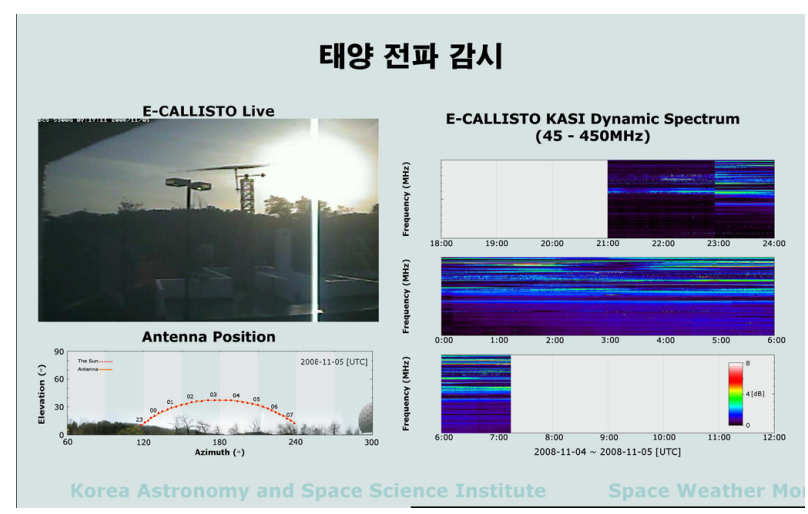

Fig. 3.- Screen view of the real time monitoring of the observation displayed in SWML. Upper left is the live image taken from the monitoring camera at the observatory. Lower left is the azimuth/elevation of the Sun and the antenna. Right is the background-subtracted dynamic spectrum.

$300 \mathrm{kHz}$. The sampling time is exactly $1.25 \mathrm{~ms}$ per frequency-pixel while the integration time is about 1 ms. The timing is controlled by a GPS clock connected via a coaxial cable. The clock gives transister-transister logic (TTL) signals at $1 \mathrm{MHz}$ with duty cycle $50 \%$.

The tracking controller, the GPS clock, and the control computer are stored inside the solar observing room for KSIS, about $30 \mathrm{~m}$ away from the antenna. The tracking controller and the GPS clock are directly connected to the tracking rotator and the spectrometer respectively via $50 \mathrm{~m}$ cables. However, CALLISTO requires Recommended Standard 232 (RS-232) serial connection to the control computer, while the maximum cable length of RS-232 is usually $15 \mathrm{~m}$. Therefore we use RS-232 to RS-422 converters at both ends so that CALLISTO and the computer can communicate each other using RS-232.

The CALLISTO application software, which was also supplied by ETH Zurich, shares the computer with the tracking control software. CALLISTO measurement time and frequency are controlled using a scheduler file and a frequency file. The Sun is expected to rise above $10^{\circ}$ after $21 \mathrm{UT}$ ( $6 \mathrm{KST}$ ) and set below before $10 \mathrm{UT}$ (19 KST) at summer solstice. Therefore the schedule is set to start at $21 \mathrm{UT}$ and end at 10 UT next day. The number of frequency channels is set to the typical value of 200 , so that the time cadence per channel is $0.25 \mathrm{~s}$. The frequencies of each channel was selected between $45 \mathrm{MHz}$ and $450 \mathrm{MHz}$ to avoid strong radio frequency interference (RFI) from artificial sources as described in $\S 3$. The detector output is read in analog-to-digital converter (ADC) digits, where $256 \mathrm{ADC}$ digits correspond to $2500 \mathrm{mV}$, and stored in a Flexible Image Transport System (FITS) file every 15 minutes. Data are stored with a scale factor and an offset applied so that the measured ADC digits range

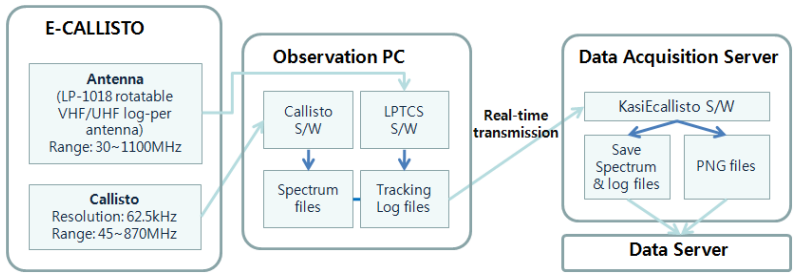

Fig. 4.- Schematic diagram of the monitoring system. Spectrum files and tracking $\log$ files produced by the CALLISTO software and tracking software (LPTCS) are transferred to the data acquisition server by the software, KasiEcallisto, which again produces PNG image files. Transferred files and image files are then stored in the data server.

fits into the byte data range $(0-255)$.

The spectrum data file and the tracking log file stored by CALLISTO software and tracking software are transferred to the data acquisition server to process and display data and the current tracking status in the Space Weather Monitoring Laboratory (SWML) in real time as shown in Fig. 3. Transferred files are processed to make image files showing dynamic spectra and antenna positions in Portable Network Graphics (PNG) format. These data and image files are again transferred to the data server which is open to the data acquisition server of ETH Zurich. Fig. 4 shows the schematic diagram of data transfer from the observation system to the data server.

\section{TEST}

\section{(a) Spectrometer Test}

Some basic tests of the spectrometer were done using a signal generator in ETH Zurich before it was carried to KASI. The measurement of the dynamic range was done at 4 different tuner-gain parameters (PWM 80, 100, 120, and 200). Fig. 5 shows the result each curve showing the output read from the detector for given input power fed from the signal generator at $150 \mathrm{MHz}$. We can find that the detector sensitivity is close to $25 \mathrm{mV} / \mathrm{dB}$ as expected. We selected the gain parameter PWM 200 for observation to maximize the sensitivity as no amplifier is used between the antenna and the spectrometer.

The spurious free dynamic range (SFDR) was measured by applying a signal with increasing power level into the low sub bands at $173 \mathrm{MHz}$. The second harmonic $(346 \mathrm{MHz})$ then falls into the frequency range of the mid band tuner and the third harmonic $(519 \mathrm{MHz})$ falls into the high band tuner. All three frequency channels were measured and stored as a function of power. As soon as the mid band or the high band tuner shows a higher response than noise floor (as a consequence to the signal applied in low band tuner) then the SFDR 


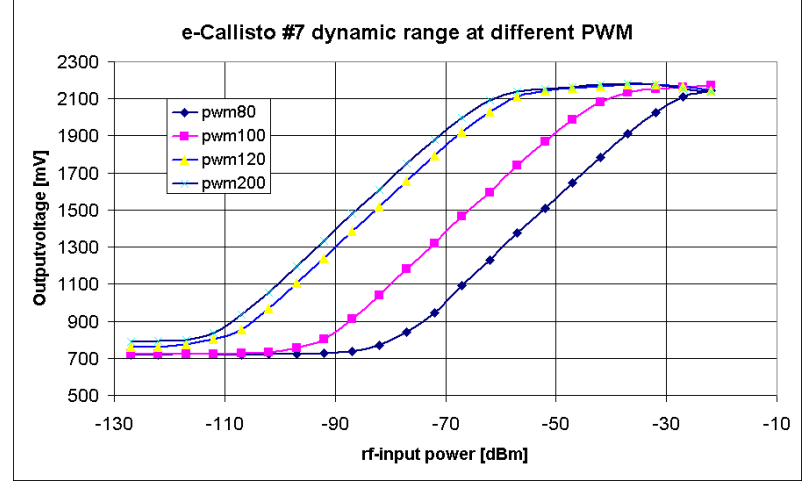

Fig. 5. - Dynamic ranges as a function of gain parameter, PWM.

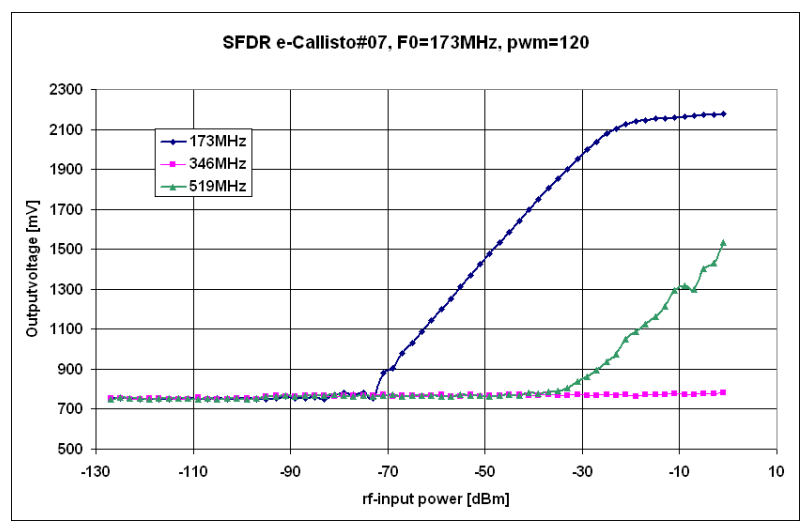

Fig. 6. - Spurious free dynamic range at the gain parameter of PWM 120. SFDR is about $40 \mathrm{~dB}$.

can be determined in dB. Fig. 6 shows the result at the gain parameter of PWM 120. The measured SFDR is about $40 \mathrm{~dB}$.

Overall bandwidth was measured by measuring the output power at a fixed frequency of $150 \mathrm{MHz}$ while the signal generator frequency was varied from $140 \mathrm{MHz}$ up to $152 \mathrm{MHz}$ in steps of $20 \mathrm{kHz}$. Fig. 7 shows the filter response plotted as a function of the applied frequency. Measured output power was normalized to the maximum value. FWHM, which corresponds to the frequency range where the attenuation is greater than $-3 \mathrm{~dB}$, is about $300 \mathrm{kHz}$.

We also calculated the Allan deviation at $607 \mathrm{MHz}$ to analyze the system noise and stability, using a free software, 'AlaVar'*. The Allan deviation is defined as the square root of the Allan variance,

$$
\sigma^{2}(\tau)=\frac{1}{2(n-1)} \sum_{i}\left(y(\tau)_{i+1}-y(\tau)_{i}\right)^{2},
$$

\footnotetext{
*http://www.alamath.com/
}

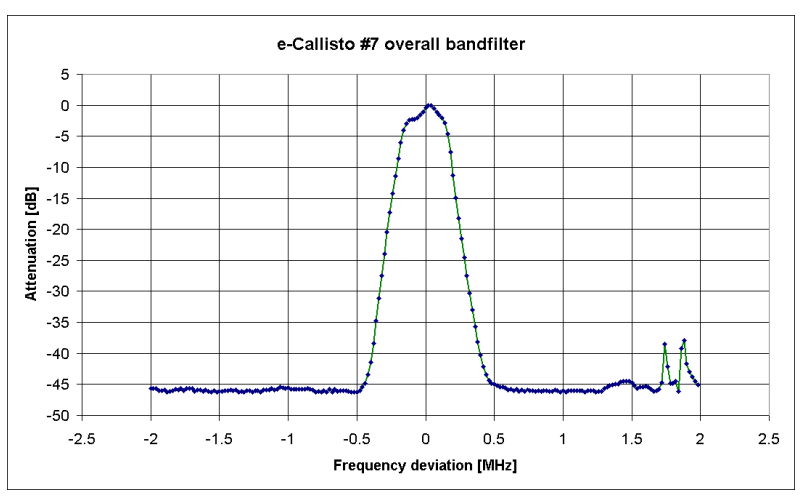

Fig. 7.- Overall band filter response at $150 \mathrm{MHz}$.

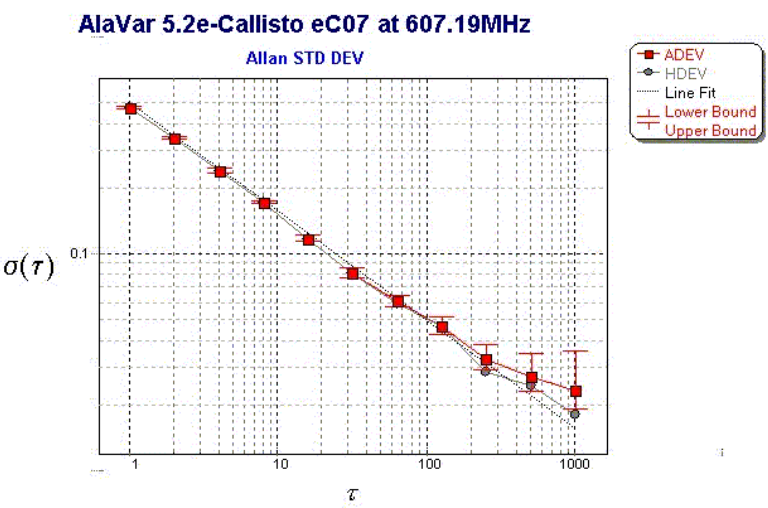

Fig. 8. - Allan deviation $\sigma(\tau)$ as a function of integration time $\tau$. Allan time here is in the order of $300 \mathrm{~s}$.

where $i$ and $n$ are the index and the number of intervals with time length $\tau$, and $y(\tau)$ is the average of the measurement in each interval. Fig. 8 shows the measured Allan deviation. It follows the characteristics of a random noise that varies in proportion to $\tau^{-1 / 2}$ up to the order of $300 \mathrm{~s}$.

\section{(b) Spectrum Measurement}

When the spectrometer was carried from ETH Zurich to KASI for installation in 2007 October, test measurements were done at KASI. The application software allows measuring whole frequency range from $45 \mathrm{MHz}$ to $870 \mathrm{MHz}$ in steps of $62.5 \mathrm{kHz}$ leading to 13120 channels output. The output is stored in a simple ASCII file in which the frequency is expressed in $\mathrm{MHz}$ and the detector output is expressed in $\mathrm{mV}$.

Approximate noise figure (NF), which describes how much noise is added by the device, was evaluated by the hot-cold measurement method using a semiconductor noise source. We measured the whole spectrum with applying a calibrated semiconductor noise source 


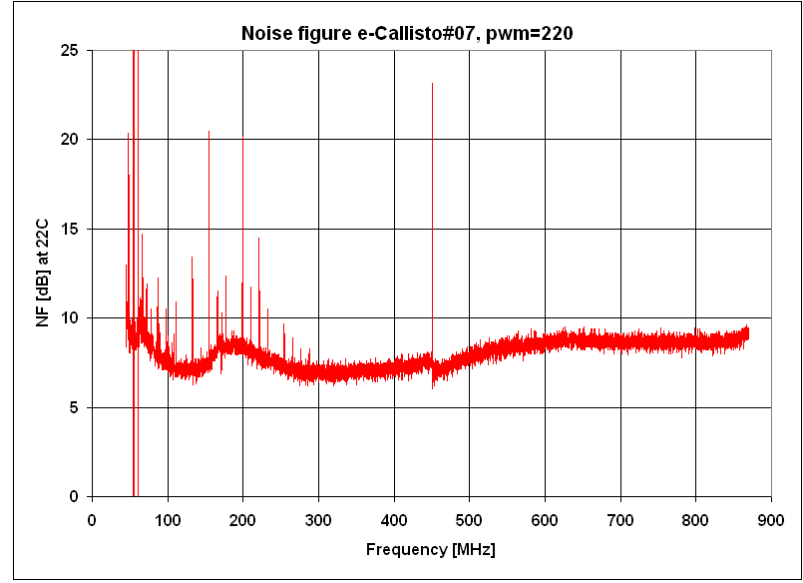

Fig. 9. - Noise figure of CALLISTO spectrometer using hot-cold method. NF on average is below $10 \mathrm{~dB}$.

attached to the spectrometer input to get a calibration signal of $14.7 \mathrm{~dB}$ ENR (Excess Noise Ratio) corresponding to about $9000 \mathrm{~K}$ excess noise relative to the ambient temperature of about $300 \mathrm{~K}$. The NF was calculated by the following approximation,

$$
N F_{d B}=E N R_{d B}-10 \log \left(10^{Y_{d B} / 10}-1\right)
$$

and Y-factor,

$$
Y_{d B}=\frac{V_{h o t}-V_{\text {cold }}}{g},
$$

where $g=25 \mathrm{mV} / \mathrm{dB}$ and $V$ is the measured voltage output when the noise source is turned on (hot) and off (cold). Fig. 9 shows the result at the gain parameter of PWM 220. The NF in average is below $10 \mathrm{~dB}$. The discontinuities around $175 \mathrm{MHz}$ and $450 \mathrm{MHz}$ are due to sub band change.

To select observation frequencies avoiding channels with terrestrial interference, we measured the whole spectrum with the fixed antenna position at south horizon. And additional measurement was taken with a $50 \Omega$ resistor applied to the antenna terminal as a reference level to evaluate the power level in $\mathrm{dB}$ above this broadband load. The measured whole spectrum is shown in Fig. 10 showing all channels in TV bands and FM band. In Daejeon, the frequency band above 450 $\mathrm{MHz}$ is already intensively used by TV and thus almost no frequency can be used for radio astronomical observation. Around $100 \mathrm{MHz}$, it is populated by very strong FM transmitters with up to $52 \mathrm{~dB}$ power level above noise floor. The situation is similar around $200 \mathrm{MHz}$ where the band is allocated to TV and digital radio broadcasting. There is also a very strong pager system at $327 \mathrm{MHz}$ with up to $50 \mathrm{~dB}$ above noise floor. We selected 200 channels between $45 \mathrm{MHz}$ and $450 \mathrm{MHz}$ for normal operation to avoid TV signal interference.

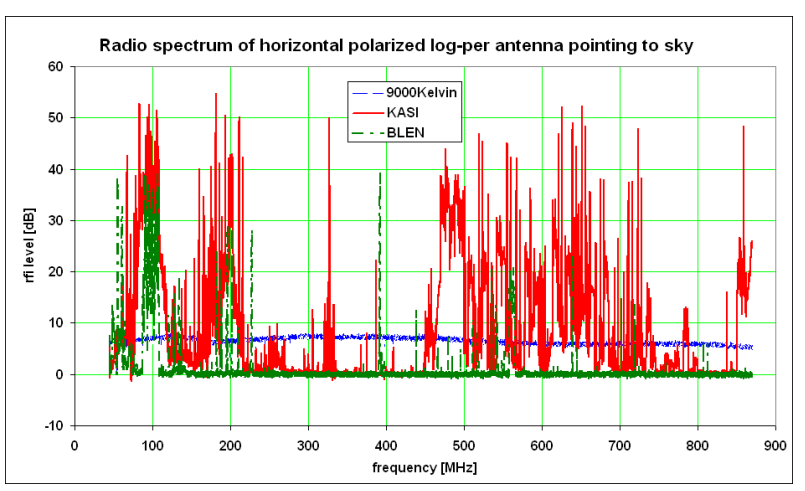

Fig. 10. - Spectral overview measured at KASI observatory. Blue line represents the Y-factor when the noise source of $9000 \mathrm{~K}$ applied. Red line and green line represent the ambient spectrum relative to the noise level $(300 \mathrm{~K})$ at KASI, Korea (red) and Bleien, Switzerland (green).

Although the Sun was not active at the time of observation, we observed the sky for several hours (see Fig. 11). This was to allow the identification of possible cross modulation by strong transmitters in the FM band and pagers systems, which may result in additional signals at non-harmonic frequencies. Only minor and sporadic cross modulation was detected during the time of observation.

\section{FIRST RESULTS}

We obtained 2 type II solar radio bursts on 2007 December 31, and found that these bursts are associated with a CME which occurred on the east limb. Fig. 12 shows the dynamic spectrum of the observed type II bursts. Mean frequency drift rates of the bursts are about $-0.1 \mathrm{MHz} / \mathrm{s}$ and $-0.07 \mathrm{MHz} / \mathrm{s}$, respectively. Inspection of Solar TErrestrial RElations Observatory (STEREO; Driesman et al. 2008) data showed that a CME occurred around 00:50 UT with a C-class flare followed and we could find no evidence of another event. This implies that two type II radio bursts resulted from one CME event, such as the cases studied by Shanmugaraju et al. (2005). The more detailed investigation of this event is currently in progress.

And we also obtained type III solar radio bursts on 2008 February 3 (Fig. 13). In this case, however, we could not find any associated flare or CME near the event time. It might be due to the short duration or weak strength of, if any, reconnection that produced the electron beam.

\section{SUMMARY AND DISCUSSION}

In collaboration with ETH Zurich, KASI successfully constructed an e-CALLISTO station in Korea at the headquarters of KASI. ETH Zurich provided the 


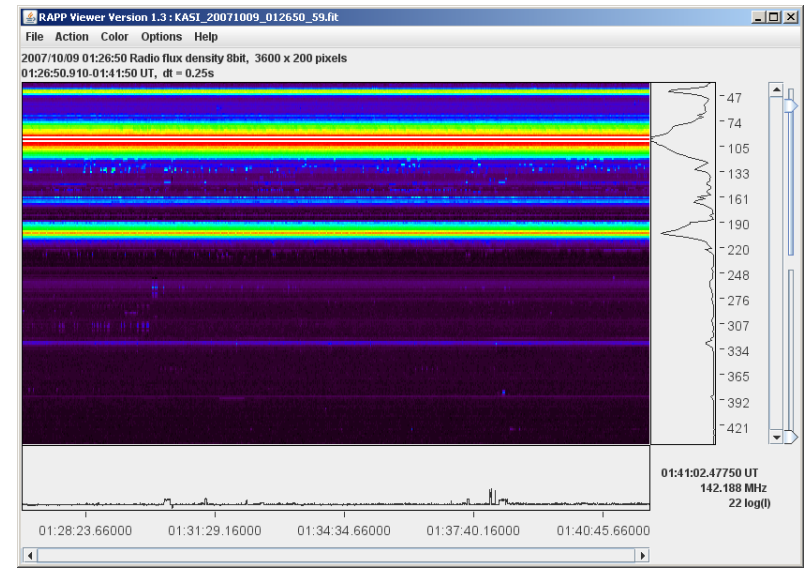

Fig. 11. - One quarter of an hour observation using CALLISTO with 200 channels per spectrum at a time resolution of $250 \mathrm{~ms}$. One can see a pile of strong carriers in FM and TV bands but no cross modulation due to too strong nearby pager transmitters.

spectrometer and KASI provided the antenna with automatic Sun tracking, computer, and GPS clock. The observation status and data are monitored in real time at SWML of KASI. The spectrometer was tested before installation to verify the performance in dynamic range, SFDR, overall bandwidth, and noise figure. After installation, spectrum of the environment was measured to select observation frequencies, and then long time observation was tested to check the cross modulation. We could detect type II radio bursts associated a CME on 2007 December 31, and several type III bursts afterward.

The spectrum of the environment showed very strong artificial signals from FM radio, pager, and TV, which may lead to cross modulation when using a high gain preamplifier. Hence Daejeon is not ideal as a host site for a solar frequency agile spectrometer to cover the whole range from $45 \mathrm{MHz}$ to $870 \mathrm{MHz}$. However, most of the frequencies between $220 \mathrm{MHz}$ and $450 \mathrm{MHz}$ except for $327 \mathrm{MHz}$ remain more or less free from interference. The first result showed the possibility that it could be used as a CME event detection system by observing below $450 \mathrm{MHz}$ with more or less acceptable interference levels.

ETH Zurich provided another CALLISTO spectrometer as a kit for KASI to combine the installation with an educational exercise. The kit was successfully assembled to verify its performance through the test. Possibly it could be used later to construct another station having better radio environment in Korea.

\section{ACKNOWLEDGEMENTS}

This work has been supported by the "Development of Korean Space Weather Center" of KASI and the
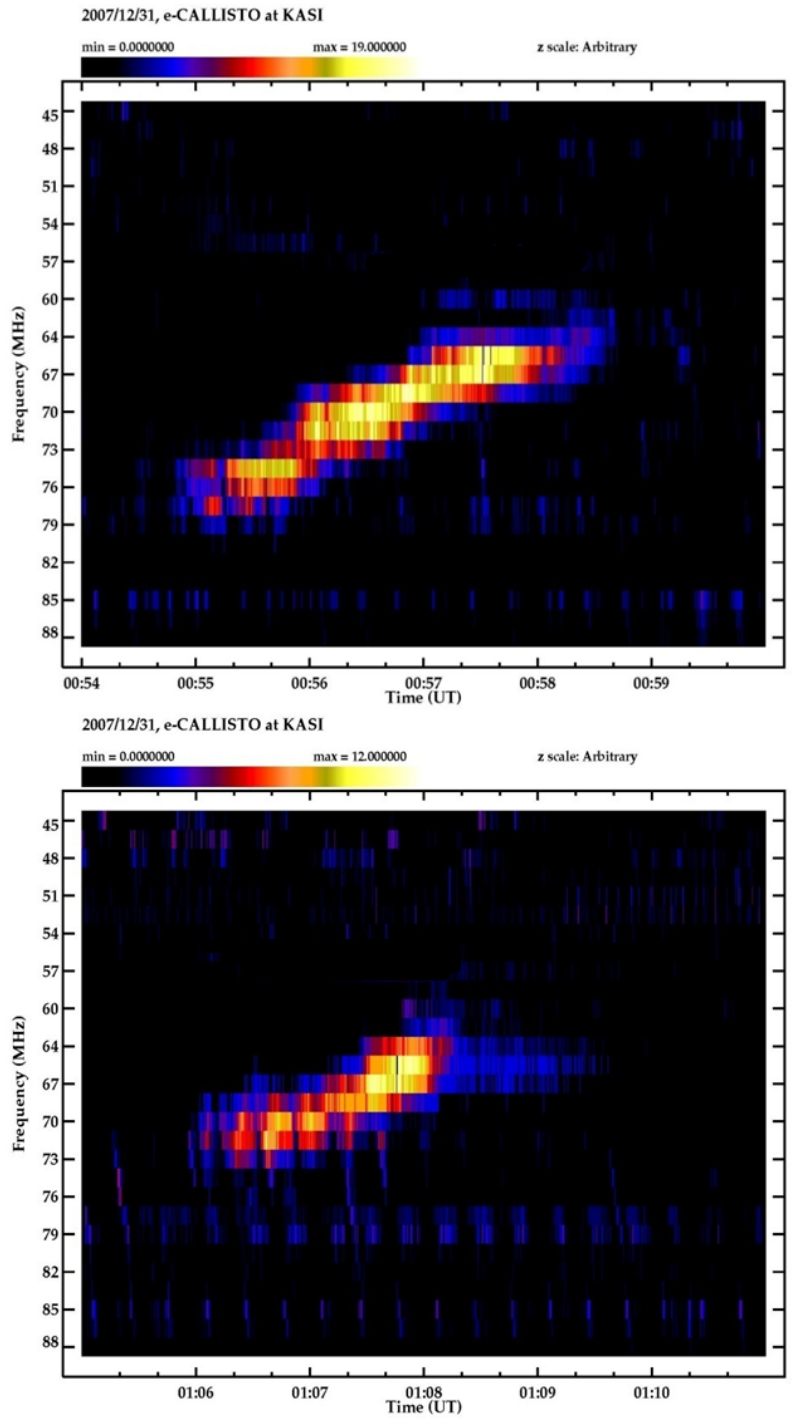

Fig. 12. - Type II radio bursts observed at 2007 December 31 00:55 UT (up) and 01:06 UT (down).

KASI basic research fund. H. R. and S. S. K. were partially supported by the Astrophysical Research Center for the Structure and Evolution of the Cosmos (ARCSEC) of the Korea Science and Engineering Foundation through the Science Research Center (SRC) program.

\section{REFERENCES}

Benz, A. O., Monstein, C., \& Meyer, H., 2005, CALLISTO - A New Concept for Solar Radio Spectrometers, Sol. Phys., 226, 143

Benz, A. O., et al., 2009, A World-wide Net of Solar Radio Spectrometers - e-CALLISTO, Earth Moon Planets, in press 


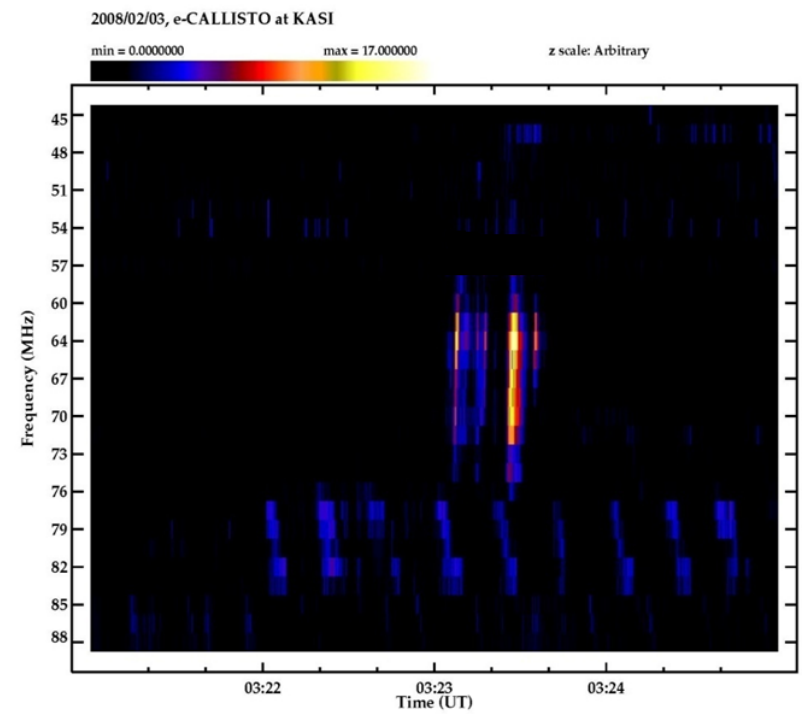

Fig. 13. - Type III radio burst observed at 2008 February 3 03:23 UT.

Cerruti, A. P., Kintner, P. M., Gary, D. E., Mannucci, A. J., Meyer, R. F., Doherty, P., \& Coster, A. J., 2008, Effect of intense December 2006 solar radio bursts on GPS receivers, Space Weather, 6, S10D07

Cho, K.-S., Bong, S.-C., Kim, Y.-H., Moon, Y.-J., Dryer, M., Shanmugaraju, A., Lee, J., \& Park, Y. D., 2008, Low coronal observations of metric type II associated CMEs by MLSO coronameters, A\&A, 491,873

Cho, K.-S., Lee, J., Gary, D. E., Moon, Y.-J., \& Park, Y. D., 2007, Magnetic Field Strength in the Solar Corona from Type II Band Splitting, ApJ, 665, 799

Cho, K.-S., Moon, Y.-J., Dryer, M., Shanmugaraju, A., Fry, C. D., Kim, Y.-H., Bong, S.-C., \& Park, Y.-D., 2005, Examination of type II origin with SOHO/LASCO observations, J. Geophys. Res., 110, A12101

Davila, J. M., Poland, A. I., \& Harrison, R. A., 2004, International heliophysical year: a program of global research continuing the tradition of previous international years, Adv. Space Res., 34, 2453

Driesman, A., Hynes, S., \& Cancro, G., 2008, The STEREO Observatory, Space Sci. Rev., 136, 17

Evans, R. M., Opher, M., Manchester, W. B., IV, \& Gombosi, T. I., 2008, Alfven Profile in the Lower Corona: Implications for Shock Formation, ApJ, 687,1355

Gary, D. E., \& Hurford, G. J., 2004, Radio Spectral Diagnostics, in Astrophys. Space Sci. Libr. Vol. 314, Solar and Space Weather Radiophysics, ed. D. E. Gary \& C. U. Keller (Dordrecht: Kluwer Academic Publishers), 71
Gopalswamy, N., Aguilar-Rodriguez, E., Yashiro, S., Nunes, S., Kaiser, M. L., \& Howard, R. A., 2005, Type II radio bursts and energetic solar eruptions, J. Geophys. Res., 110, A12S07

Hey, J. S., 1946, Solar radiations in the 4-6 meter radio wavelength band, Nature, 158, 234

Isliker, H. \& Benz, A. O., 1994, Catalogue of 1-3 GHz solar flare radio emission, A\&AS, 104, 145

Kim, Y.-H., Moon, Y.-J., Cho, K.-S., Park, Y.-D., Choi, S.-H., Jang, B.-H., \& Kim, S., 2006, Development of KASI Solar Imaging Spectrograph, Publ. Korean Astron. Soc., 21, 51

Lanzerotti, L. J., 2004, Solar and Solar Radio Effects on Technologies, in Astrophys. Space Sci. Libr. Vol. 314, Solar and Space Weather Radiophysics, ed. D. E. Gary \& C. U. Keller (Dordrecht: Kluwer Academic Publishers), 1

Roh, D.-G. \& Jung, J. H., 1999, Characteristics of TRAO 14m Radio Telescope (1999), Publ. Korean Astron. Soc., 14, 123

Shanmugaraju, A., Moon, Y.-J., Cho, K.-S., Kim, Y.H., Dryer, M., \& Umapath, S., 2005, Multiple Type II Solar Radio Bursts, Sol. Phys., 232, 87

Shen, C., Wang, Y., Ye, P., Zhao, X. P., Gui, B., \& Wang, S., 2007, Strength of Coronal Mass Ejectiondriven Shocks near the Sun and Their Importance in Predicting Solar Energetic Particle Events, ApJ, 670,849

Wild, J. P., 1985, The Beginnings, in Solar Radiophysics, ed. D. J. McLean \& N. R. Labrum (Cambridge: Cambridge University Press), 3 